\title{
Computing the feedback filter of the decision feedback equalizer at the FFT speed
}

\author{
A. Spalvieri and M. Magarini \\ Dipartimento di Elettronica e Informazione, Politecnico di Milano, \\ Piazza Leonardo da Vinci, 32, I-20133 Milano (Italy), \\ \{spalvier,magarini\}@elet.polimi.it
}

\begin{abstract}
This paper is devoted to the efficient computation of the feedback and feedforward filters of the decision feedback equalizer. The key operation in the computation of the filters is the spectral factorization. The paper proposes to adopt the efficient method for spectral factorization due to Kolmogoroff, which runs at the speed of FFT. Since the duration in the time domain of the impulse response obtained from the spectral factorization is not under the control of the designer, truncation of that impulse response becomes necessary. We propose a method that mitigates the performance loss due to the truncation, and validate the proposal by computer simulation on a variety of channels.
\end{abstract}

\section{INTRODUCTION}

Decision feedback equalization is a simple and effective technique for detection of signals affected by noise and InterSymbol Interference (ISI). The Decision Feedback Equalizer (DFE) consists of a FeedForward Filter (FFF), an instantaneous detector, and a FeedBack Filter (FBF) that operates cancellation of the ISI due to the past data by feeding back a weighted combination of the past decisions taken by the detector. The most popular DFE dates back to Monsen [1]. In the DFE of Monsen, which is known as the Minimum Mean Square Error DFE (MMSE-DFE), the FFF and the FBF optimize the Mean Square Error (MSE) between the input to the detector and the transmitted constellation symbol. Note that it has been recently observed in [2] that the FFF and the FBF of the MMSE-DFE allow maximum likelihood sequence detection with minimum number of states, and that they can also be adopted in suboptimal sequence detection schemes such as the delayed decision feedback sequence detector of [3]. Although the scope of the present paper is limited to decision feedback equalization, it is clear that the observation of [2] broadens the applicability of the MMSE-DFE FFF and FBF, thus encouraging interest to the very classical research topic of efficient computation of the two filters.

Computation of the FFF and FBF by methods that operate entirely in the time domain has been widely studied in the past. The idea behind this class of methods is to model the channel and both filters in the FIR form, and to optimize the coefficients of the two filters. It often happens in the practice that the number of taps of the FFF is greater than the number of taps of the channel and of the FBF. In this case, the complexity of the computation is dominated by the computation of the FFF which goes with $n^{2}$, where $n$ is the number of taps of the FIR FFF (see [4], [5]).
The increasing use in practical transmission systems of block transmission with intensive block-based processing, has recently stimulated interest to the computation and the realization of the FFF in the discrete frequency domain, thus taking advantage from the efficiency of the FFT algorithm. Specifically, a method that is based on time-domain computation of the FBF and frequency-domain computation of the FFF can be derived from the approach of Belfiore and Park [6], and has been recently proposed by Falconer et al. [7] and by Benvenuto and Tomasin [8]. This method optimizes the coefficients of the FIR FBF by applying the Levinson recursion, which computes the coefficients of the FBF with a complexity that goes with the square of the number of coefficients. The FFF is computed in the discrete frequency domain by sampling the optimal transfer function, thus exploiting the efficiency of the FFT/IFFT algorithm. Since it often happens that the number of taps of the FBF is much lower than the number of points of the FFT, this method leads to more efficient computation than the class of methods based on computation of the FFF in the time domain.

It is known that the key operation in the computation of the DFE feedback filter is the spectral factorization, and that it is also known that the method due to Kolmogoroff, which belongs to the class of cepstral methods, computes the spectral factorization by making use only of FFTs and look-up tables (see e.g. [9]). When block processing making use of FFT is adopted, the computation of the feedback filter based on FFT becomes attractive, because often the speed of the FFT is so large that one can reuse the FFT kernel more times in the time taken by the transmission of one block. The possibility of performing the spectral factorization by cepstral methods is mentioned in [10] in the context of equalization, but it is not further pursed in that paper. One potential limit to the application of the Kolmogoroff method is that no constraints can be imposed on the duration of the impulse response of the FBF. Therefore the number of taps of the FBF obtained by the Kolmogoroff method depends only on the duration of the impulse response of the channel, which, in the wireless scenario, is not under the control of the designer. This is potentially a severe drawback, because, in the practice, what one can do is either to design a DFE with a large number of feedback taps or to truncate the impulse response of the FBF. The large number of feedback taps would sharpen the problem of error propagation, and, moreover, it would also lead to reduced spectral efficiency in the block transmission 
proposed in [7], [8], which is based on a cyclic prefix of duration equal to the number of taps of the FBF. Truncation of the impulse response of the FBF would lead to degradation of the error performance. The issue of mitigating the performance loss due to truncation was considered by Qureshi and Newhall in the context of shortening methods for suboptimal sequence detection [11]. The sequence detector with reduced number of states of [11] uses in the Viterbi algorithm a truncated version of the impulse response of the FBF of the zero-forcing DFE. The performance loss due to truncation is mitigated by filtering the signal fed to the sequence detector through the MMSE feedforward filter.

We observe that the shortening method proposed by Qureshi and Newhall for the sequence detector is not well suited to shorten the impulse response of the FBF of the DFE. As a matter of fact, after truncation, the impulse response of the FBF could loose the minimum phase property, leading to dramatic performance loss. Note that the minimum phase property is much more important for the DFE than for the sequence detector. Henceforth, we introduce a variation to the method of Qureshi and Newhall that makes the minimum phase property robust against truncation. Specifically, we propose of designing the FBF from a deliberately biased version of the power spectrum of signal plus noise. More precisely, a constant term, which is called virtual noise, is added to the true power spectrum. The biased spectrum is factored, then the inverse Fourier transform of the spectral factor is truncated to the desired duration.

The rest of the paper is organized as follows. In Section II we present the system model and the background material for decision feedback equalization. In Section III computation of the spectral factor by the FFT algorithm and truncation of the impulse response of the spectral factor are presented. In section IV experimental results are given for a static AWGN channel and for two multipath fading channels. In section $\mathrm{V}$ conclusions are drawn.

\section{BACKGROUND}

\section{A. System Model}

We adopt the discrete time Additive White Gaussian Noise (AWGN) baseband equivalent model of the observation, and use the $z$-transform to represent sequences $\left(z^{-1}\right.$ indicates the unit delay). Let

$$
X(z)=\sum_{k} x_{k} z^{-k}=A(z) H(z)+W(z)
$$

be the $z$-transform of the observation, where all the quantities are assumed to be complex-valued. In the above equation, $H(z)$ is the $z$-transform of the impulse response of the channel, $A(z)$ represents the i.i.d. data sequence, and $W(z)$ represents the AWGN. Data are drawn from an $(M-P A M)^{m}$ constellation, $m=1,2$ with variance

$$
\sigma_{a}^{2}=m \frac{M^{2}-1}{3}
$$

In the following, we denote by $\Psi_{s}(z)$ the $z$-transform of the autocorrelation of the sequence represented by $S(z)$, being understood that $\Psi_{s}(z)=S(z) S^{*}\left(z^{-1}\right)=\sum_{k} z^{-k} \sum_{i} s_{i}^{*} s_{i+k}$ when $\sum_{i}\left|s_{i}\right|^{2}<\infty$, while $\Psi_{s}(z)=\sum_{k} z^{-k} E\left\{s_{i}^{*} s_{i+k}\right\}$ when $S(z)$ represents a stationary random sequence. In the above notation, the superscript ${ }^{*}$ denotes the complex conjugate. The $z$-transform of the autocorrelation of the AWGN is

$$
\Psi_{w}(z)=N_{0}
$$

The $z$-transform of the autocorrelation of the observation is

$$
\Psi_{x}(z)=\sigma_{a}^{2} H(z) H^{*}\left(z^{-1}\right)+N_{0} .
$$

The Signal-to-Noise Ratio (SNR) is :

$$
\mathrm{SNR}=\frac{\sigma_{a}^{2} \sum_{i}\left|h_{i}\right|^{2}}{N_{0}} .
$$

\section{B. $M S E-D F E$}

Aiming to frequency-domain computation of the FFF, it is convenient to derive the MSE-DFE feedforward and feedback filters by the approach of predictive DFE developed by Belfiore and Park [6]. Let the $z$-transform of the error at the decision device be

$V(z)=(1-B(z))(C(z) X(z)-A(z))=D(z)(C(z) X(z)-A(z))$,

where $B(z)$ is the $z$-transform of the impulse response of the FBF and $C(z)$ represents the FFF of the predictive DFE. The term

$$
U(z)=C(z) X(z)-A(z)
$$

is the error that is predicted by the FBF. The MSE is

$J=\frac{T}{2 \pi} \int_{-\pi / T}^{\pi / T} \Psi_{v}\left(e^{j \omega T}\right) d \omega=\frac{T}{2 \pi} \int_{-\pi / T}^{\pi / T} \Psi_{d}\left(e^{j \omega T}\right) \Psi_{u}\left(e^{j \omega T}\right) d \omega$.

Since $\Psi_{d}\left(e^{j \omega T}\right) \geq 0$ and $\Psi_{u}\left(e^{j \omega T}\right) \geq 0$, one concludes that the optimal FFF minimizes frequency-by-frequency the power spectral density of the error before the linear prediction, independently of the FBF. Therefore the optimal FFF is the MMSE linear equalizer, that is

$$
C(z)=\frac{\sigma_{a}^{2} H^{*}\left(z^{-1}\right)}{\Psi_{x}(z)} .
$$

With the MMSE linear equalizer one has

$$
\Psi_{u}(z)=\frac{\sigma_{a}^{2} N_{0}}{\Psi_{x}(z)}
$$

and

$$
\Psi_{v}(z)=\frac{\sigma_{a}^{2} N_{0} \Psi_{d}(z)}{\Psi_{x}(z)} .
$$

The computation of the FBF proceeds as follows. We wish to predict $u_{k}$ by linear prediction from the past samples $u_{k-1}, u_{k-2}, \ldots$, assuming that they are known. Let

$$
v_{k}=u_{k}-\sum_{i=1}^{\mu} b_{i} u_{k-i}
$$

be the error after linear prediction with $\mu$ taps. The coefficients $\mathbf{b}=\left(b_{1}, b_{2}, \ldots, b_{\mu}\right)$ are obtained as

$$
\mathbf{b}=\arg \min _{\mathbf{b}} E\left\{\left|v_{k}\right|^{2}\right\} .
$$




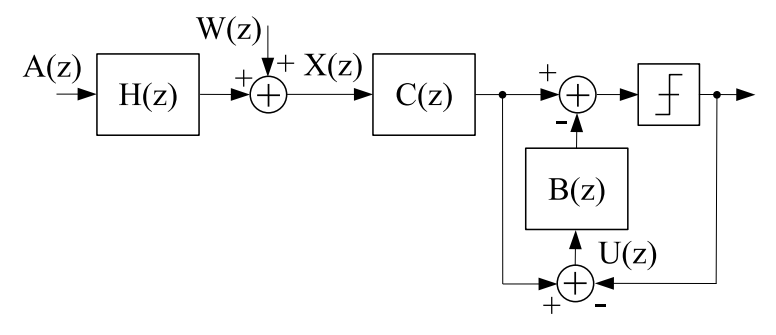

Fig. 1. Block diagram of the system.

The unique minimum is obtained by putting to zero the partial derivatives of the power of the error with respect to the entries of $\mathbf{b}$ :

$$
\begin{aligned}
\frac{\partial}{\partial b_{i}} E\left\{\left|v_{k}\right|^{2}\right\} & =-E\left\{v_{k} u_{k-i}^{*}\right\}=-E\left\{\left(u_{k}-\sum_{j=1}^{\mu} b_{j} u_{k-j}\right) u_{k-i}^{*}\right\} \\
& =-\psi_{u, i}+\sum_{j=1}^{\mu} b_{j} \psi_{u, i-j}=0, \quad i=1,2, \ldots, \mu
\end{aligned}
$$

where $\psi_{u, i}$ is the $i$-th sample of the autocorrelation of the sequence represented by $U(z)$. Hence the taps of the predictor are obtained by solving the system of linear equations

$$
\sum_{j=1}^{\mu} b_{j} \psi_{u, i-j}=\psi_{u, i}, \quad i=1,2, \ldots, \mu,
$$

which are known as the Yule-Walker equations. The $D(z)$ obtained from the Yule-Walker equations as $D(z)=1-B(z)$ is monic $\left(d_{0}=1\right)$, causal, and minimum phase (all the zeros of $D(z)$ are on or inside the unit circle). The block diagram of the system is reported in Fig. 1, where, as it happens in practical systems, the available decisions are used in place of the true transmitted data sequence.

Computation of the FFF in the frequency domain requires only frequency-by-frequency inversion of $\Psi_{x}\left(e^{j \omega T}\right)$ and frequency-by-frequency multiplication between the inverse of the power spectrum and the matched filter. Note that inversion of $\Psi_{x}\left(e^{j \omega T}\right)$ is also required in the computation of the FBF. After the above mentioned inversion, the computation of the two filters is performed by two parallel branches, which can be an advantage in VLSI implementation. Computation of the FBF with $\mu$ taps by the Levinson recursion has a complexity that goes with $\mu^{2}$. The conventional (nonpredictive) MSE-DFE with ideal FFF is obtained by using the cascade of linear MMSE and $D(z)$ as FFF, and by feeding back through $B(z)$ the past data.

\section{MMSE-DFE}

Ideal prediction is obtained when the power spectrum of the error after prediction is white, leading to the MMSE-DFE. We write the white power spectrum of the error after prediction as the fraction

$$
\Psi_{v}(z)=\frac{\sigma_{a}^{2} N_{0}}{\alpha} .
$$

The scalar $\alpha$ can be computed as [12]:

$$
\alpha=\exp \left(\frac{T}{2 \pi} \int_{-\pi / T}^{\pi / T} \log \left(\Psi_{x}\left(e^{j \omega T}\right)\right) d \omega\right) .
$$

For the MSE of the MMSE-DFE one gets

$$
J=\sigma_{a}^{2} N_{0} \alpha^{-1}=\sigma_{a}^{2} N_{0} \exp \left(-\frac{T}{2 \pi} \int_{-\pi / T}^{\pi / T} \log \left(\Psi_{x}\left(e^{j \omega T}\right)\right) d \omega\right) .
$$

Note that for the MMSE-DFE one has

$$
\Psi_{v}(z)=\frac{\sigma_{a}^{2} N_{0} D(z) D^{*}\left(z^{-1}\right)}{\Psi_{x}(z)}=\frac{\sigma_{a}^{2} N_{0}}{\alpha},
$$

which demonstrates that $D(z)$ is obtained from the spectral factorization

$$
\alpha D(z) D^{*}\left(z^{-1}\right)=\Psi_{x}(z),
$$

where the $D(z)$ that is monic, causal, and minimum phase, is taken. When $\Psi_{v}(z)$ is FIR with $2 \nu+1$ taps, the FBF of the MMSE-DFE has $\nu$ taps.

\section{Computing FBF AT THE SpeEd OF FFT}

The most efficient method known for the computation of the spectral factorization is the method due to Kolmogoroff [9]:

$$
\sqrt{\alpha} D\left(e^{j \omega T}\right)=\exp \left(\operatorname{FT}\left(u s_{k} \odot \operatorname{IFT}\left(\log \left(\Psi_{x}\left(e^{j \omega T}\right)\right)\right)\right)\right),
$$

where FT indicates the Fourier Transform, IFT indicates the Inverse Fourier Transform, $\left\{u s_{k}\right\}$ is the unit step sequence, and $\odot$ is the Shur-Hadamard (elementwise) product. In the literature of signal processing the term $\log \left(\Psi_{x}\left(e^{j \omega T}\right)\right)$ takes the name of cepstrum. The computation requires only Fourier transformations and the log-exp operation, which can be implemented by look-up table. Passing from the FT to the FFT one achieves the complexity $\mathcal{O}(n \log (n))$, where $n$ is the number of points of the FFT.

As mentioned in the introduction, the duration of the impulse response of the spectral factor resulting from (1) depends only on the duration of the impulse response of the channel, which, in the wireless scenario, it is not under the control of the designer. Truncation of the impulse response could induce dramatic performance loss because the truncated spectral factor could loose the minimum phase property. Experimentally one can observe that the minimum phase property is often lost when $\Psi_{x}(z)$ has zeros that are close to the unit circle. Therefore one would like to bring these zeros far from the unit circle before the factorization of the power spectrum. One way to achieve this objective is to perform the following spectral factorization

$$
\alpha D(z) D^{*}\left(z^{-1}\right)=\Psi_{x}(z)+\lambda,
$$

where $\lambda \geq 0$ is up to the designer. The parameter $\lambda$ adds a floor to the power spectrum, thus preventing the presence of spectral zeros. Note that as $\lambda \rightarrow \infty$, the feedback taps of the DFE tend to zero, and the receiver is reduced to the pure MMSE linear equalizer. After the spectral factorization and the IFFT, only the first $\mu+1$ taps of the impulse response are retained. Unfortunately, it is difficult to derive by analytical means the optimal $\lambda$ for the given channel and for the desired $\mu$. What one can do is to apply the procedure for many values of $\lambda$ and evaluate the MSE as

$$
J=\frac{T \sigma_{a}^{2}}{2 \pi N_{0}} \int_{-\pi / T}^{\pi / T} \frac{D_{T}\left(e^{j \omega T}\right) D_{T}^{*}\left(e^{-j \omega T}\right)}{\Psi_{x}\left(e^{j \omega T}\right)} d \omega,
$$




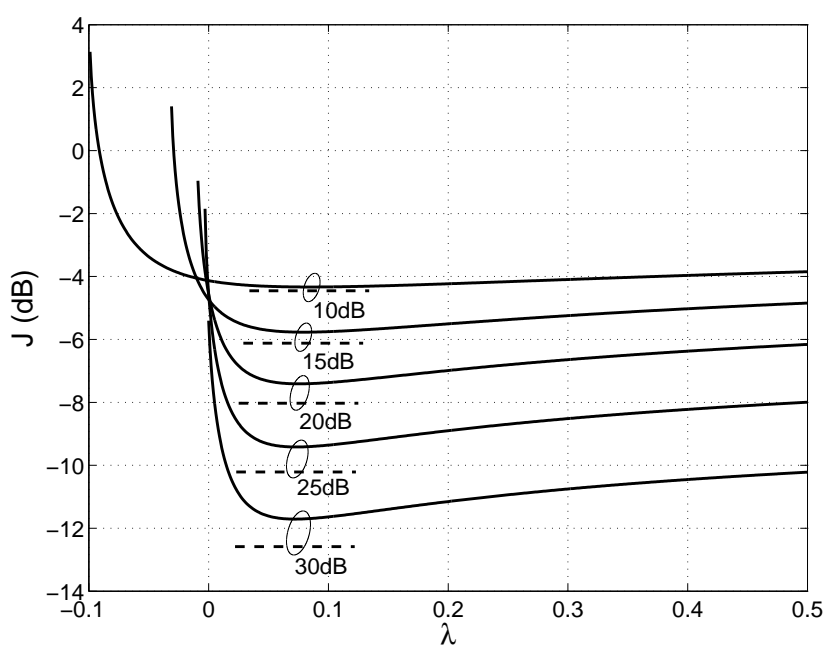

Fig. 2. MSE versus $\lambda$ for the AWGN channel with the truncation method for memory of the FBF equal to 4. Curves are parametric in the SNR that is reported next to them. The dashed lines represent the MSE that is obtained when the coefficients of the FBF are computed by the Yule-Walker equations.

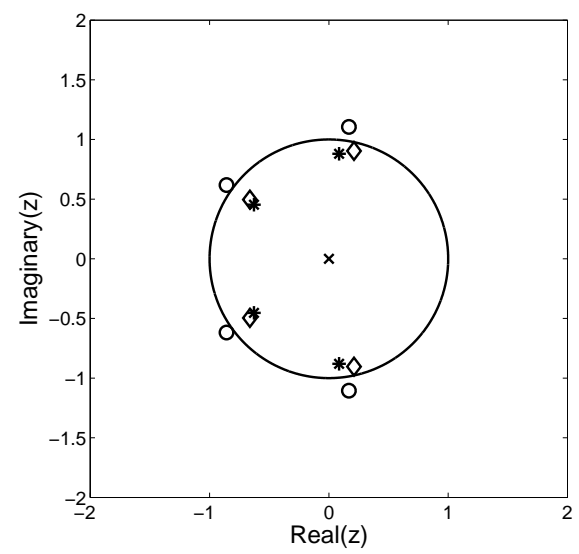

Fig. 3. Zeros of the impulse responses of the FBF obtained by the application of the competitor methods at $\mathrm{SNR}=20 \mathrm{~dB}$. Truncation with $\lambda=0$ : 'o'; Truncation with $\lambda=0.08$ : ' $\diamond$; Yule-Walker equations: '*'.

where $D_{T}(z)$ is the truncated spectral factor. This allows to select the best $\lambda$ for the given channel and the desired $\mu$.

\section{EXPERIMENTAL RESULTS}

The benefits of introducing $\lambda$ in (2) to obtain robust truncation of the spectral factor are demonstrated on a variety of channels. In this section we report examples of AWGN and multipath fading channels. In the following we assume that the channel is always perfectly known at the receiver. For all the examples the spectral factor was computed by performing the FFT over 512 points. As a first example we report simulation results for the AWGN channel $H(z)=0.176+0.316 z^{-1}+$ $0.476 z^{-2}+0.532 z^{-3}+0.476 z^{-4}+0.316 z^{-5}+0.176 z^{-6}$. It is worth noting that this channel is the worst channel with $\nu=6$ [14]. Simulation results for this channel were carried out by setting the memory of the FBF to $\mu=4$ and by considering BPSK modulation. As mentioned in the previous section, the preliminary operation of selecting the optimal $\lambda$ is required. In Fig. 2 it is reported the MSE versus $\lambda$ computed according to equation (3). From the figure it

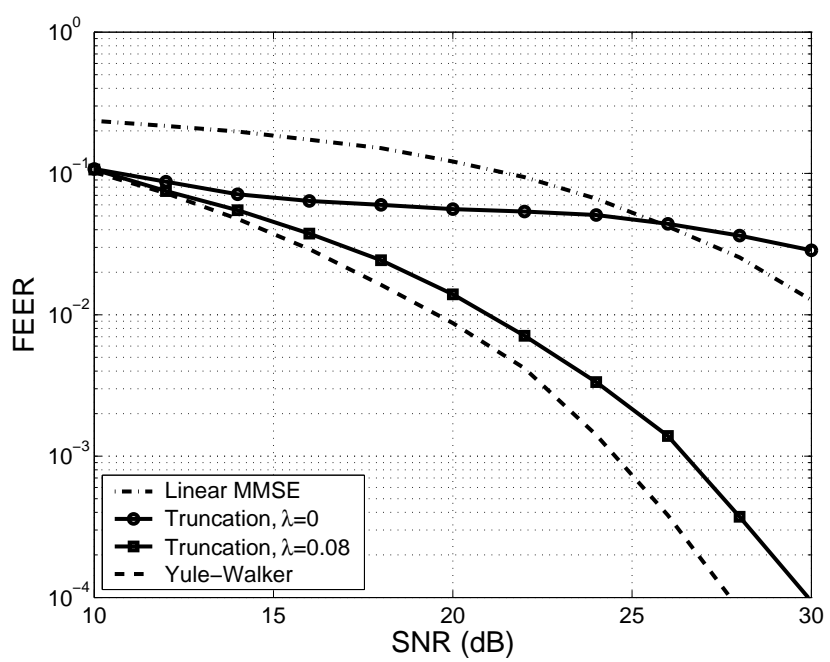

Fig. 4. FEER versus SNR for the AWGN channel with memory of the FBF equal to 4 .

can be observed that the MSE is a smooth function of $\lambda$, which is a desirable property because the performance is not much sensitive to the choice of $\lambda$. It can be seen from the figure that good performance is obtained for $\lambda=0.08$ at any SNR. We observe that, for $\lambda=0$, the MSE obtained by the truncation method is insensitive to the SNR, so that at high SNR the performance is dramatically degraded, making the truncation method not suitable. Conversely, a judicious choice of $\lambda$ brings the performance of the truncation method near to the performance of the MSE-DFE at any SNR. Fig. 3 reports the zeros of the impulse responses obtained by the application of different methods for $\mathrm{SNR}=20 \mathrm{~dB}$. Note that the bad performance obtained for $\lambda=0$ by the truncation method is explained by observing that this method gives a maximum phase FBF (all the roots are outside the unit circle), while the minimum phase property of the FBF is preserved by adding the bias. Fig. 4 shows the First Error Event Rate (FEER) versus SNR of the truncation method for $\lambda=0$ and $\lambda=0.08$. The FEER is measured by a random sequence of $10^{6}$ symbols. The performance of the Yule-Walker equations and of the linear MMSE is also reported. The figure confirms the observations made on Fig. 2 regarding the effectiveness of the bias.

As far as the multipath fading channel is concerned we consider two examples. The first is the channel model A defined by ETSI BRAN for HiperLAN/2 [15]. The modulation scheme that we consider is QPSK. In this case simulations are used to generate $10^{7}$ realizations of the fading channel. For each channel realization we compute the output Signalto-Disturbance Ratio (SDR) of the MMSE-DFE as

$$
\mathrm{SDR}=\frac{\sigma_{a}^{2}-J}{J} .
$$

The optimal $\lambda$ is obtained by optimizing the average SDR. The instantaneous FEER for QPSK is given by

$$
\text { FEER } \approx 2 Q\left(\sqrt{\frac{\mathrm{SDR}}{2 \sigma_{a}^{2}}}\right),
$$




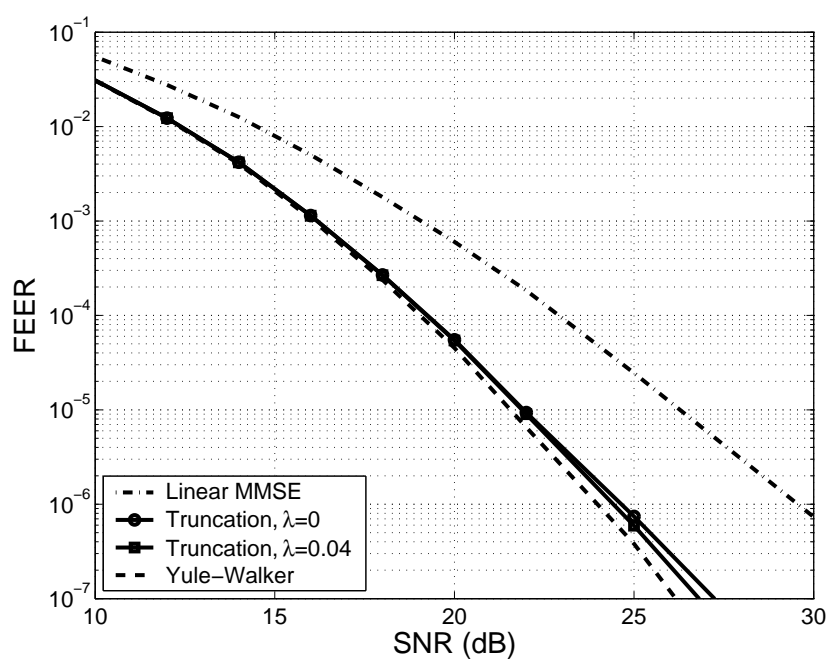

Fig. 5. FEER versus SNR for the Hiperlan type A channel with memory of the FBF equal to 3 .

where

$$
Q(x)=\frac{1}{2 \pi} \int_{x}^{\infty} e^{-\frac{u^{2}}{2}} d u .
$$

Note that to compute the FEER one should derive the probability density function of ISI plus noise, for example by the method proposed in [13]. However, a fairly accurate estimate is obtained by considering the Gaussian approximation. The FEER reported in the figures is obtained by averaging the instantaneous FEER over the $10^{7}$ channel realizations. Fig. 5 shows the performance for $\mu=3$ of the proposed method. We observe that, with the fading channel, the performance of the truncation method is close to the performance of the MSE-DFE, and that it is not much sensitive to the bias. A small improvement can be obtained by adding the bias only at high SNR. At FEER $=10^{-7}$ the performance of the proposed method with $\lambda=0.04$ is approximately $0.5 \mathrm{~dB}$ better than with $\lambda=0$ and $0.9 \mathrm{~dB}$ worse than that of the Yule-Walker equations. The second example of multipath fading channel was obtained by considering each coefficient of the AWGN channel of [14] as a Rayleigh random variable with average power equal to the square of the corresponding coefficient. In order to increase the time duration of the channel we inserted 3 zeros in between each coefficient. Fig. 6 shows the performance of the competitor methods for $\mu=7$. Also in this case we note that the performance of the truncation method is not much sensitive to the bias, and that it is close to the performance of the MSE-DFE. Small differences are appreciated only at high SNR. At FEER $=10^{-7}$ the performance of the truncation method with $\lambda=0.1$ is approximately 1.2 $\mathrm{dB}$ better than with $\lambda=0$ and $1.1 \mathrm{~dB}$ worse than that of the Yule-Walker equations.

\section{CONCLUSIONS}

Efficient computation of the forward and backward filters of the decision feedback equalizer is possible by computing the spectral factorization by the method due to Kolmogoroff. The disadvantage of the method is that the time duration of the feedback filter is not under control, therefore, in the practice,

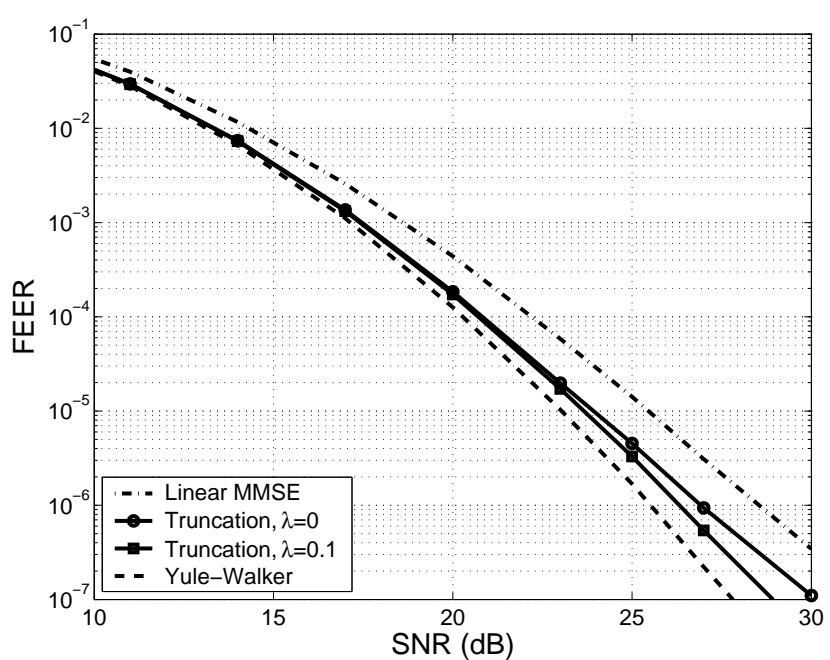

Fig. 6. FEER versus SNR for the second example of multipath fading channel with memory of the FBF equal to 7 .

truncation becomes necessary. We have proposed to mitigate the performance loss due to truncation by adding a bias in the factored power spectrum. Experimental results obtained with a severe static channel and with two fading channels show that our proposal makes the performance of the truncated DFE close to the performance of the DFE with feedback filter obtained from the Yule-Walker equations.

\section{REFERENCES}

[1] P. Monsen, "Feedback equalization for fading dispersive channels," IEEE Trans. Inform. Theory, vol. 17, pp. 56-64, Jan. 1971.

[2] M. Magarini, A. Spalvieri, G. Tartara, "The mean-square delayed decision feedback sequence detector," IEEE Trans. Commun., vol. 50, pp. 1462-1470, Sept. 2002.

[3] A. Duel-Hallen, C. Heegard, "Delayed decision feedback sequence estimation,” IEEE Trans. Commun., vol. 37, pp. 428-436, May 1989.

[4] N. Al-Dhahir, J. Cioffi, "MMSE decision-feedback equalizers: finitelength results," IEEE Trans. Inform. Theory, vol. 41, pp. 961-975, July 1995.

[5] N. Al-Dhahir, J. Cioffi, "Fast computation of channel-estimate based equalizers in packet data transmission," IEEE Trans. Signal Proc., vol. 43, pp. 2462-2473, Nov. 1995.

[6] C. Belfiore, J. Park, "Decision feedback equalization," Proc. IEEE, vol. 67, pp. 1143-1156, Aug. 1979.

[7] D. Falconer, S. L. Ariyavisitakul, A. Benyamin-Seeyar, B. Eidson, "Frequency domain equalization for single-carrier broadband wireless systems," IEEE Commun. Mag., vol. 40 , pp. 58-66, Apr. 2002.

[8] N. Benvenuto, S. Tomasin, "On the comparison between OFDM and single carrier modulation with a DFE using a frequency-domain feedforward filter," IEEE Trans. Commun., vol. 50, pp. 947-955, June 2002.

[9] J. Claerbout. Fundamentals of geophysical data processing. [Online]. Available: www.sep.stanford.edu/sep/prof/.

[10] W. H. Gerstacker, F. Obernosterer, R. Meyer, J. B. Huber, "On prefilter computation for reduced-state equalization," IEEE Trans. Wireless Commun., vol. 1, pp. 793-800, Oct. 2002.

[11] S. U. H. Qureshi, E. E. Newhall, "An adaptive receiver for data transmission aver time-dispersive channels," IEEE Trans. Inform. Theory, vol. 19, pp. 448-457, July 1973.

[12] J. Salz, "Optimum mean-square decision feedback equalization," Bell Syst. Tech. J., vol. 52, pp. 1341-1373, Oct. 1973.

[13] J. C. Vanelli, N. M. Shehadeh, "Computation of bit error probability using the trapezoidal integration rule," IEEE Trans. Commun., vol. 22, pp. 331-334, March 1974.

[14] R. R. Anderson, G. J. Foschini, "The minimum distance for MLSE digital data systems of limited complexity," IEEE Trans. Inform. Theory, vol. 21 , pp. 544-551, Sept. 1975.

[15] J. Medbo, P. Schramm, "Channel models for HiperLAN2," ETSI/BRAN document no. 3ERI085B, 1998. 